TRANSACTIONS OF THE

AMERICAN MATHEMATICAL SOCIETY

Volume 363, Number 2, February 2011, Pages 901-921

S 0002-9947(2010)05141-4

Article electronically published on September 21, 2010

\title{
QUANTUM ISOMETRY GROUPS OF 0-DIMENSIONAL MANIFOLDS
}

\author{
JYOTISHMAN BHOWMICK, DEBASHISH GOSWAMI, AND ADAM SKALSKI
}

\begin{abstract}
Quantum isometry groups of spectral triples associated with approximately finite-dimensional $C^{*}$-algebras are shown to arise as inductive limits of quantum symmetry groups of corresponding truncated Bratteli diagrams. This is used to determine explicitly the quantum isometry group of the natural spectral triple on the algebra of continuous functions on the middlethird Cantor set. It is also shown that the quantum symmetry groups of finite graphs or metric spaces coincide with the quantum isometry groups of the corresponding classical objects equipped with natural Laplacians.
\end{abstract}

\section{INTRODUCTION}

Following the pioneering ideas of Wang described in Wan (and motivated by Connes), a number of mathematicians including Bichon, Banica and others (Bic, Ban ) have defined and studied the universal objects in certain categories of quantum groups, all of which are quantum generalizations of groups acting on (typically finite) sets or algebras preserving some given underlying structure such as a metric or a functional. In this way, they have come up with several universal (compact) quantum groups corresponding to the classical group of permutations (more generally, isometries w.r.t. a given metric) of a finite set, the group of symmetries of a finite graph or the automorphism group of a finite-dimensional matrix algebra. Motivated by their work, the first two authors of the present article have begun a systematic effort to define and study similar universal quantum groups beyond the finite-dimensional or 'discrete' setup, more precisely, in the framework of (possibly noncommutative) differential geometry as proposed by Connes $\left(\mathrm{Co}_{2}\right)$. They have been able to formulate a quantum group analogue of the group of Riemannian isometries (Gos, $\left.\mathrm{BG}_{1}\right)$ as well as the group of orientation-preserving isometries $\left(\mathrm{BG}_{2}\right)$ of a (possibly noncommutative, given by spectral triple) Riemannian spin manifold. Many well-known and important compact quantum groups (e.g. $S O_{q}(3)$ ) have been identified with such universal quantum groups for some suitably chosen spectral triples.

Received by the editors August 1, 2008 and, in revised form, May 7, 2009.

2010 Mathematics Subject Classification. Primary 58B32; Secondary 81R50, 81R60, 46L87.

Key words and phrases. Compact quantum group, quantum isometry groups, spectral triples, $A F$ algebras.

(C)2010 American Mathematical Society Reverts to public domain 28 years from publication 
Recently in $\mathrm{CI}_{1}$ and $\mathrm{CI}_{2}$, Christensen and Ivan constructed natural spectral triples on approximately finite-dimensional $(A F) C^{*}$-algebras. The starting point for this article is the desire to understand and compute quantum isometry groups of resulting noncommutative manifolds. $A F$ algebras provide a natural 'connecting bridge' between the finite- and infinite-dimensional noncommutative spaces and thus can be thought of as 0-dimensional manifolds. This is reinforced by the fact that Christensen and Ivan showed that on each $A F$ algebra one can construct spectral triples with arbitrarily good summability properties. We show that the 'quantum group of orientation-preserving isometries' of a Christensen-Ivan type triple arises as an inductive limit of quantum isometry groups of certain finitedimensional triples (Theorem 1.2). In the case when the $A F$ algebra in question is commutative, the resulting quantum isometry groups of relevant finite-dimensional objects fit into the framework described at the beginning of the Introduction, as we show that they coincide with quantum symmetry groups of finite graphs obtained by suitable truncations of the Bratteli diagrams. This observation implies that the construction we consider can be thought of as giving a definition of a quantum symmetry group of an arbitrary Bratteli diagram. It also enables us to compute explicitly the quantum isometry group of a spectral triple associated with the middle-third Cantor set introduced first by Connes and later studied by Christensen and Ivan. As a by-product of our considerations of this example we see that contrary to the classical case a quantum isometry of the product set preserving the first factor in the suitable sense need not be a product isometry.

Having determined the universal objects for actions on 0-dimensional noncommutative manifolds, it is natural to look back and see how one can accommodate the already existing theory of quantum permutation and quantum automorphism groups of 'finite' structures in the more general setup of quantum isometry groups. This is the second of the main objectives of the present article. We have been able to identify the quantum group of automorphisms of a finite metric space or a finite graph in the sense of Banica and Bichon with the quantum group of orientation (and suitable 'volume-form') preserving isometries of a natural spectral triple, thus successfully unifying the approaches of $[\mathrm{Ban}]$ and $[\mathrm{Bic}]$ with that of $\overline{\mathrm{Gos}}]$ and $\mathrm{BG}_{1}$. We finish the paper by suggesting a possible approach to defining quantum isometric actions on general (compact) metric spaces and compute two explicit examples of universal quantum groups of 'isometries' in such a context.

The detailed plan of the article is as follows: we begin by introducing basic notation and recalling fundamental concepts related to quantum groups of (orientationpreserving) isometries, as defined in $\mathrm{Gos}$ ] and $\mathrm{BG}_{2}$. Section 1 contains a description of the limit construction for an inductive system of compact quantum groups and its application for quantum isometry groups. In Section 2 we recall the construction of spectral triples on $A F$ algebras due to Christensen and Ivan, show basic properties of the compact quantum groups appearing in the related inductive system and relate them for commutative $A F$ algebras with the quantum symmetry groups of truncated Bratteli diagrams. This is used to compute in Section 3 the quantum isometry group for Connes's spectral triple related to the Cantor set. In Section 4 it is shown that the quantum symmetry group of a finite metric space $X$ ( $\mathrm{Ban}]$ ) coincides with the quantum isometry group resulting from equipping the algebra of functions on $X$ with a natural Laplacian; the result has a natural variant for the quantum symmetry group of a finite graph. Finally Section 5 contains a 
suggestion of a tentative definition of quantum isometry of a general metric space $X$ and computation of such an object for $X=[0,1]$ and $X=S^{1}$.

\section{NOTATION AND PRELIMINARIES}

The symbol $\otimes$ will always denote the minimal/spatial tensor product of $C^{*}$ algebras; the purely algebraic tensor product will be denoted by $\odot$. We will occasionally use the language of Hilbert $C^{*}$-modules and multiplier algebras (see Lan). Often when $X$ is a finite set we will write $C(X)$ to denote the algebra of all complex functions on $X$, with the point of view that when $X$ becomes an infinite topological space the correct generalisation is the algebra of continuous functions on $X$.

A compact quantum group (c.q.g.) is a pair $(\mathrm{S}, \Delta)$, where $\mathrm{S}$ is a unital separable $C^{*}$-algebra and $\Delta: \mathrm{S} \rightarrow \mathrm{S} \otimes \mathrm{S}$ is a unital $C^{*}$-homomorphism satisfying the coassociativity:

(ai) $(\Delta \otimes$ id $) \circ \Delta=($ id $\otimes \Delta) \circ \Delta$ and the quantum cancellation properties:

(aii) the linear spans of $\Delta(\mathrm{S})(\mathrm{S} \otimes 1)$ and $\Delta(\mathrm{S})(1 \otimes \mathrm{S})$ are norm-dense in $\mathrm{S} \otimes \mathrm{S}$.

Occasionally we will simply call $\mathrm{S}$ a compact quantum group, understanding by this the existence of a suitable coproduct $\Delta$ on S. By a morphism in the category of compact quantum groups we understand a unital ${ }^{*}$-homomorphism intertwining the respective coproducts.

A c.q.g. (S, $\Delta)$ is said to (co)-act on a unital $C^{*}$-algebra $\mathrm{A}$ if there is a unital $C^{*}$ homomorphism (called an action) $\alpha: \mathrm{A} \rightarrow \mathrm{A} \otimes \mathrm{S}$ satisfying the following conditions:

(bi) $(\alpha \otimes$ id $) \circ \alpha=($ id $\otimes \Delta) \circ \alpha$,

(bii) the linear span of $\alpha(\mathrm{A})(1 \otimes \mathrm{S})$ is norm-dense in $\mathrm{A} \otimes \mathrm{S}$.

A unitary (co-) representation of a compact quantum group $(\mathrm{S}, \Delta)$ on a Hilbert space $\mathrm{H}$ is a linear map $U$ from $\mathrm{H}$ to the $C^{*}$-Hilbert $\mathrm{S}$-module $\mathrm{H} \otimes \mathrm{S}$ such that the element $\widetilde{U} \in \mathcal{M}(\mathcal{K}(\mathrm{H}) \otimes \mathrm{S})$ given by the formula $\widetilde{U}(\xi \otimes b)=U(\xi)(1 \otimes b)(\xi \in \mathrm{H}, b \in \mathrm{S}))$ is a unitary satisfying

$$
(\mathrm{id} \otimes \Delta) \widetilde{U}=\widetilde{U}_{(12)} \widetilde{U}_{(13)} .
$$

In the last formula we used the standard 'leg' notation: for an operator $X \in \mathcal{B}\left(\mathrm{H}_{1} \otimes\right.$ $\left.\mathrm{H}_{2}\right), X_{(12)}$ and $X_{(13)}$ denote respectively the operators $X \otimes I_{\mathrm{H}_{2}} \in \mathcal{B}\left(\mathrm{H}_{1} \otimes \mathrm{H}_{2} \otimes \mathrm{H}_{2}\right)$ and $\sigma_{23} X_{12} \sigma_{23} \in \mathcal{B}\left(\mathrm{H}_{1} \otimes \mathrm{H}_{2} \otimes \mathrm{H}_{2}\right)\left(\sigma_{23}\right.$ being the unitary on $\mathrm{H}_{1} \otimes \mathrm{H}_{2} \otimes \mathrm{H}_{2}$ which flips the two copies of $\mathrm{H}_{2}$ ).

Given a unitary representation $U$ of $(\mathrm{S}, \Delta)$ we denote by $\alpha_{U}$ the *-homomorphism $\alpha_{U}(X)=\widetilde{U}(X \otimes 1) \widetilde{U}^{*}$ for $X \in \mathcal{B}(\mathrm{H})$. If $\tau$ is a not necessarily bounded, but densely (in the weak operator topology) defined linear functional on $\mathcal{B}(\mathrm{H})$, we say that $\alpha_{U}$ preserves $\tau$ if $\alpha_{U}$ maps a suitable weakly dense ${ }^{*}$-subalgebra (say $\mathcal{D}$ ) in the domain of $\tau$ into $\mathcal{D} \odot \mathrm{S}$ and $(\tau \otimes \mathrm{id})\left(\alpha_{U}(a)\right)=\tau(a) 1_{\mathrm{S}}$ for all $a \in \mathcal{D}$. When $\tau$ is bounded and normal, this is equivalent to the condition $(\tau \otimes \mathrm{id})\left(\alpha_{U}(a)\right)=\tau(a) 1_{\mathrm{S}}$ being satisfied by all $a \in \mathcal{B}(\mathrm{H})$.

We say that a (possibly unbounded) operator $T$ on $\mathrm{H}$ commutes with $U$ if $T \otimes I$ (with the natural domain) commutes with $\widetilde{U}$. Sometimes such an operator will be called $U$-equivariant.

We briefly recall the definitions of quantum isometry groups, referring to Gos and $\overline{\mathrm{BG}_{2}}$ for the details. Let $\left(\mathcal{A}^{\infty}, \mathrm{H}, D\right)$ be a spectral triple (of compact type, see $\mathrm{Co}_{1}$; note however that as we will often consider here finite-dimensional objects we $d o$ not require $D$ to be unbounded). Consider the category $\mathbf{Q}^{\prime}$ whose objects are pairs $(\widetilde{\mathrm{S}}, U)$, where $\widetilde{\mathrm{S}}$ is a compact quantum group and $U$ is a unitary representation 
of $\widetilde{\mathrm{S}}$ in $\mathrm{H}$ such that the action $\alpha_{U}$ maps $\mathcal{A}^{\infty}$ into the ampliation of its weak closure, and moreover $U$ commutes with $D$. The set of morphisms $\operatorname{Mor}\left((\mathrm{S}, U),\left(\mathrm{S}^{\prime}, U^{\prime}\right)\right)$ is the set of c.q.g. morphisms $\Phi: S \rightarrow S^{\prime}$ satisfying the condition (id $\left.\otimes \Phi\right)(U)=U^{\prime}$. If, additionally, we are given a (possibly unbounded) positive operator $R$ such that $R$ commutes with $D$, we also consider the subcategory $\mathbf{Q}_{R}^{\prime}$ of $\mathbf{Q}^{\prime}$ consisting of the S-actions for which $\alpha_{U}$ preserves the functional $\tau_{R}$ as in $\mathrm{BG}_{2}$. It is proved in $\mathrm{BG}_{2}$ that $\mathbf{Q}_{R}^{\prime}$ has a universal object, denoted by $\widetilde{\mathrm{QISO}}_{R}^{+}\left(\mathcal{A}^{\infty}, \mathrm{H}, D\right)$ or simply $\widetilde{\mathrm{QISO}}_{R}^{+}(D)$. We shall denote by $\mathrm{QISO}_{R}^{+}(D)$ the Woronowicz subalgebra of $\widetilde{\mathrm{QISO}}_{R}^{+}(D)$ such that $\alpha_{U}$ faithfully maps $\mathcal{A}^{\infty}$ into $\left(\mathcal{A}^{\infty}\right)^{\prime \prime} \otimes \mathrm{QISO}_{R}^{+}(D)$, and this subalgebra is called the quantum group of (R-twisted) volume and orientation-preserving isometries of the underlying spectral triple. It is also proved in $\mathrm{BG}_{2}$, Theorem 2.14] that under some further conditions a universal object in the bigger category $\mathbf{Q}^{\prime}$ exists. It is denoted by $\widetilde{\mathrm{QISO}}^{+}(D)$. The corresponding Woronowicz subalgebra for which $\left.\alpha_{U}\right|_{\mathcal{A}^{\infty}}$ is faithful is denoted by QISO $^{+}(D)$ and called the quantum group of orientationpreserving isometries.

\section{INDUCTIVE LIMIT CONSTRUCTION FOR QUANTUM ISOMETRY GROUPS}

In this section we describe the limiting construction for an inductive system of compact quantum groups and give an application for quantum isometry groups which is fundamental for the results of the next section.

The following lemma is probably known, but we include the proof for the sake of completeness.

Lemma 1.1. Suppose that $\left(\mathrm{S}_{n}\right)_{n \in \mathbb{N}}$ is a sequence of compact quantum groups and for each $n, m \in \mathbb{N}, n \leq m$ there is a c.q.g. morphism $\pi_{n, m}: \mathrm{S}_{n} \rightarrow \mathrm{S}_{m}$ with the compatibility property

$$
\pi_{m, k} \circ \pi_{n, m}=\pi_{n, k}, \quad n \leq m \leq k .
$$

Then the inductive limit of $C^{*}$-algebras $\left(\mathrm{S}_{n}\right)_{n \in \mathbb{N}}$ has a canonical structure of a compact quantum group. It will be denoted by $\mathrm{S}_{\infty}$ or $\lim _{n \in \mathbb{N}} \mathrm{S}_{n}$. It has the following universality property:

for any c.q.g. (S, $\Delta$ ) such that there are c.q.g. morphisms $\pi_{n}: \mathrm{S}_{n} \rightarrow \mathrm{S}$ satisfying for all $m, n \in \mathbb{N}, m \geq n$ the equality $\pi_{m} \circ \pi_{n, m}=\pi_{n}$, there exists a unique c.q.g. morphism $\pi_{\infty}: \mathrm{S}_{\infty} \rightarrow \mathrm{S}$ such that $\pi_{n}=\pi_{\infty} \circ \pi_{n, \infty}$ for all $n \in \mathbb{N}$, where we have denoted by $\pi_{n, \infty}$ the canonical unital $C^{*}$-homomorphism from $\mathrm{S}_{n}$ into $\mathrm{S}_{\infty}$.

Proof. Let us denote the coproduct on $\mathrm{S}_{n}$ by $\Delta_{n}$. We consider the unital $C^{*}$ homomorphism $\rho_{n}: \mathrm{S}_{n} \rightarrow \mathrm{S}_{\infty} \otimes \mathrm{S}_{\infty}$ given by $\rho_{n}=\left(\pi_{n, \infty} \otimes \pi_{n, \infty}\right) \circ \Delta_{n}$ and observe that these maps do satisfy the compatibility property:

$$
\rho_{m} \circ \pi_{n, m}=\rho_{n} \forall n \leq m
$$

Thus, by the general properties of the $C^{*}$-algebraic inductive limit, we have a unique unital $C^{*}$-homomorphism $\Delta_{\infty}: \mathrm{S}_{\infty} \rightarrow \mathrm{S}_{\infty} \otimes \mathrm{S}_{\infty}$ satisfying $\Delta_{\infty} \circ \pi_{n, \infty}=\rho_{n}$ for all $n$. We claim that $\left(\mathrm{S}_{\infty}, \Delta_{\infty}\right)$ is a c.q.g.

We first check that $\Delta_{\infty}$ is coassociative. It is enough to verify the coassociativity on the dense set $\bigcup_{n} \pi_{n, \infty}\left(\mathrm{S}_{n}\right)$. Indeed, for $s=\pi_{n, \infty}(a)\left(a \in \mathrm{S}_{n}\right)$, by using $\Delta_{\infty} \circ$ 
$\pi_{n, \infty}=\left(\pi_{n, \infty} \otimes \pi_{n, \infty}\right) \circ \Delta_{n}$, we have the following:

$$
\begin{aligned}
\left(\Delta_{\infty} \otimes \mathrm{id}\right) \Delta_{\infty}\left(\pi_{n, \infty}(a)\right) & =\left(\Delta_{\infty} \otimes \mathrm{id}\right)\left(\pi_{n, \infty} \otimes \pi_{n, \infty}\right)\left(\Delta_{n}(a)\right) \\
& =\left(\pi_{n, \infty} \otimes \pi_{n, \infty} \otimes \pi_{n, \infty}\right)\left(\Delta_{n} \otimes \mathrm{id}\right)\left(\Delta_{n}(a)\right) \\
& =\left(\pi_{n, \infty} \otimes \pi_{n, \infty} \otimes \pi_{n, \infty}\right)\left(\mathrm{id} \otimes \Delta_{n}\right)\left(\Delta_{n}(a)\right) \\
& =\left(\pi_{n, \infty} \otimes\left(\pi_{n, \infty} \otimes \pi_{n, \infty}\right) \circ \Delta_{n}\right)\left(\Delta_{n}(a)\right) \\
& =\left(\pi_{n, \infty} \otimes \Delta_{\infty} \circ \pi_{n, \infty}\right)\left(\Delta_{n}(a)\right) \\
& =\left(\mathrm{id} \otimes \Delta_{\infty}\right)\left(\left(\pi_{n, \infty} \otimes \pi_{n, \infty}\right)\left(\Delta_{n}(a)\right)\right) \\
& =\left(\mathrm{id} \otimes \Delta_{\infty}\right)\left(\Delta_{\infty}\left(\pi_{n, \infty}(a)\right)\right),
\end{aligned}
$$

which proves the coassociativity.

Finally, we need to verify the quantum cancellation properties. Note that to show that $\Delta_{\infty}\left(S_{\infty}\right)\left(1 \otimes S_{\infty}\right)$ is dense in $S_{\infty} \otimes S_{\infty}$ it is enough to show that the above assertion is true with $S_{\infty}$ replaced by a dense subalgebra $\bigcup_{n} \pi_{n, \infty}\left(S_{n}\right)$.

Using the density of $\Delta_{n}\left(\mathrm{~S}_{n}\right)\left(1 \otimes \mathrm{S}_{n}\right)$ in $\mathrm{S}_{n} \otimes \mathrm{S}_{n}$ and the contractivity of the map $\pi_{n, \infty}$ we note that $\left(\pi_{n, \infty} \otimes \pi_{n, \infty}\right)\left(\Delta_{n}\left(S_{n}\right)\left(1 \otimes \mathrm{S}_{n}\right)\right)$ is dense in $\left(\pi_{n, \infty} \otimes \pi_{n, \infty}\right)\left(\mathrm{S}_{n} \otimes \mathrm{S}_{n}\right)$. This implies that $\left(\pi_{n, \infty} \otimes \pi_{n, \infty}\right)\left(\Delta_{n}\left(\mathrm{~S}_{n}\right)\right)\left(1 \otimes \pi_{n, \infty}\left(\mathrm{S}_{n}\right)\right)$ is dense in $\pi_{n, \infty}\left(\mathrm{S}_{n}\right) \otimes$ $\pi_{n, \infty}\left(\mathrm{S}_{n}\right)$ and hence $\Delta_{\infty}\left(\pi_{n, \infty}\left(\mathrm{S}_{n}\right)\right)\left(1 \otimes \pi_{n, \infty}\left(\mathrm{S}_{n}\right)\right)$ is dense in $\pi_{n, \infty}\left(\mathrm{S}_{n}\right) \otimes \pi_{n, \infty}\left(\mathrm{S}_{n}\right)$. The proof of the claim now follows by noting that $\pi_{n, \infty}\left(\mathrm{S}_{n}\right)=\pi_{m, \infty} \pi_{n, m}\left(\mathrm{~S}_{n}\right) \subseteq$ $\pi_{m, \infty}\left(\mathrm{S}_{m}\right)$ for any $m \geq n$, along with the above observations. The right quantum cancellation property can be shown in the same way.

The proof of the universality property is routine and hence omitted.

Note that the proof remains valid for any other indexing set for the net, not necessarily $\mathbb{N}$.

The next theorem connects the inductive construction above with some specific quantum isometry groups.

Theorem 1.2. Suppose that $\mathrm{A}$ is a $C^{*}$-algebra acting on a Hilbert space $\mathrm{H}$ and that $D$ is a (densely defined) selfadjoint operator on $\mathrm{H}$ with compact resolvent, such that $D$ has a one-dimensional eigenspace spanned by a vector $\xi$ which is cyclic and separating for $\mathrm{A}$. Let $\left(\mathcal{A}_{n}\right)_{n \in \mathbb{N}}$ be an increasing net of unital ${ }^{*}$-subalgebras of $\mathrm{A}$ and put $\mathcal{A}=\bigcup_{n \in \mathbb{N}} \mathcal{A}_{n}$. Suppose that $\mathcal{A}$ is dense in $\mathrm{A}$ and that for each $a \in \mathcal{A}$ the commutator $[D, a]$ is densely defined and bounded. Additionally put $\mathrm{H}_{n}=\overline{\mathcal{A}_{n} \xi}$, let $P_{n}$ denote the orthogonal projection on $\mathrm{H}_{n}$ and assume that each $P_{n}$ commutes with $D$. Then each $\left(\mathcal{A}_{n}, \mathrm{H}_{n},\left.D\right|_{\mathrm{H}_{n}}\right)$ is a spectral triple satisfying the conditions of Theorem 2.14 of $\mathrm{BG}_{2}$ and there exist natural compatible c.q.g. morphisms $\pi_{m, n}$ :

$$
\begin{gathered}
{\widetilde{\mathrm{QISO}^{+}}}^{+}\left(\mathcal{A}_{m}, \mathrm{H}_{m},\left.D\right|_{\mathrm{H}_{m}}\right) \rightarrow \widetilde{\mathrm{QISO}}^{+}\left(\mathcal{A}_{n}, \mathrm{H}_{n},\left.D\right|_{\mathrm{H}_{n}}\right)(n, m \in \mathbb{N}, m \leq n) \text { and } \\
\widetilde{\operatorname{QISO}}^{+}(\mathcal{A}, \mathrm{H}, D)=\lim _{n \in \mathbb{N}} \widetilde{\mathrm{QISO}}^{+}\left(\mathcal{A}_{n}, \mathrm{H}_{n},\left.D\right|_{\mathrm{H}_{n}}\right) .
\end{gathered}
$$

Similar conclusions hold if we replace everywhere above $\widetilde{\mathrm{QISO}}^{+}$by $\mathrm{QISO}^{+}$.

Proof. We prove the assertion corresponding to $\widetilde{\mathrm{QISO}}^{+}$only, since the proof for $\mathrm{QISO}^{+}$follows by very similar arguments. Let us denote $\widetilde{\mathrm{QISO}^{+}}\left(\mathcal{A}_{n}, \mathrm{H}_{n}, D_{n}\right)$ by $\mathrm{S}_{n}$ and the corresponding unitary representation $\left(\right.$ in $\left.\mathrm{H}_{n}\right)$ by $U_{n}$. Let us denote the category of compact quantum groups acting by orientation-preserving isometries on $\left(\mathcal{A}_{n}, \mathrm{H}_{n},\left.D\right|_{\mathrm{H}_{n}}\right)$ and $(\mathcal{A}, \mathrm{H}, D)$ respectively by $\mathbf{C}_{\mathbf{n}}$ and $\mathbf{C}$. 
Since $U_{n}$ is a unitary which commutes with $\left.D_{n} \equiv D\right|_{\mathrm{H}_{n}}$ and hence preserves the eigenspaces of $D_{n}$, it restricts to a unitary representation of $S_{n}$ on each $H_{m}$ for $m \leq n$. In other words, $\left(\mathrm{S}_{n},\left.U_{n}\right|_{\mathbf{H}_{m}}\right) \in \operatorname{Obj}\left(\mathbf{C}_{\mathbf{m}}\right)$, and by the universality of $\mathbf{S}_{m}$ there exists a compact quantum group morphism, say, $\pi_{m, n}: \mathrm{S}_{m} \rightarrow \mathrm{S}_{n}$ such that $\left.\left(\mathrm{id} \otimes \pi_{m, n}\right) U_{m}\right|_{\mathrm{H}_{m}}=\left.U_{n}\right|_{\mathrm{H}_{m}}$.

Let $p \leq m \leq n$. Then we have $\left.\left(\mathrm{id} \otimes \pi_{m, n} \pi_{p, m}\right) U_{p}\right|_{\mathbf{H}_{p}}=\left.U_{n}\right|_{\mathbf{H}_{p}}$. It follows by the uniqueness of the map $\pi_{p, n}$ that $\pi_{p, n}=\pi_{m, n} \pi_{p, m}$; i.e. $\left(\mathrm{S}_{n}\right)_{n \in \mathbb{N}}$ forms an inductive system of compact quantum groups satisfying the assumptions of Lemma 1.1. Denote by $\mathrm{S}_{\infty}$ the inductive limit c.q.g. obtained in that lemma, with $\pi_{n, \infty}: \mathrm{S}_{n} \rightarrow \mathrm{S}$ denoting the corresponding c.q.g. morphisms. The family of formulas $\left.U\right|_{\mathrm{H}_{n}}:=$ (id $\left.\otimes \pi_{n, \infty}\right) \circ U_{n}$ combine to define a unitary representation $U$ of $\mathrm{S}_{\infty}$ on $\mathrm{H}$. It is also easy to see from the construction that $U$ commutes with $D$. This means that $\left(\mathrm{S}_{\infty}, U\right) \in \operatorname{Obj}(\mathbf{C})$; hence there exists a unique surjective c.q.g. morphism from $\mathrm{S}:=\widetilde{\mathrm{QISO}}^{+}(\mathcal{A}, \mathrm{H}, D)$ to $\mathrm{S}_{\infty}$ identifying $\mathrm{S}_{\infty}$ as a quantum subgroup of $\mathrm{S}$.

The proof will now be complete if we can show that there is a surjective c.q.g. morphism in the reverse direction, identifying $S$ as a quantum subgroup of $S_{\infty}$. This can be deduced from Lemma 1.1 by using the universality property of the inductive limit. Indeed, for each $n \in \mathbb{N}$ the unitary representation, say $V_{n}$, of $\widetilde{\mathrm{QISO}^{+}}(\mathcal{A}, \mathrm{H}, D)$ restricts to $\mathrm{H}_{n}$ and commutes with $D$ on that subspace, thus inducing a c.q.g. morphism $\rho_{n}$ from $\mathrm{S}_{n}=\widetilde{\mathrm{QISO}}^{+}\left(\mathcal{A}_{n}, \mathrm{H}_{n}, D_{n}\right)$ into $\mathrm{S}$. The family of morphisms $\left(\rho_{n}\right)_{n \in \mathbb{N}}$ satisfies the compatibility conditions required in Lemma 1.1. It remains to show that the induced c.q.g. morphism $\rho_{\infty}$ from $\mathrm{S}_{\infty}$ into $\mathrm{S}$ is surjective. By the faithfulness of the representation $V$ of $\widetilde{\mathrm{QISO}}^{+}(\mathcal{A}, \mathrm{H}, D)$, we know that the span of matrix elements corresponding to all $V_{n}$ forms a norm-dense subset of $\mathrm{S}$. As the range of $\rho_{n}$ contains the matrix elements corresponding to $V_{n}=\left.V\right|_{\mathbf{H}_{n}}$, the proof of surjectivity of $\rho_{\infty}$ is finished.

The assumptions of the theorem might seem very restrictive. In the next section however we will describe a natural family of spectral triples on $A F$ algebras, constructed in $\mathrm{CI}_{1}$, for which we have exactly the situation as above.

\section{QUANTUM ISOMETRY GROUPS FOR SPECTRAL TRIPLES ON $A F$ ALGEBRAS}

We first recall the construction of natural spectral triples on $A F$ algebras due to E. Christensen and C. Ivan $\left(\mathrm{CI}_{1}\right)$. Let A be a unital $A F C^{*}$-algebra, the norm closure of an increasing sequence $\left(\mathrm{A}_{n}\right)_{n \in \mathbb{N}}$ of finite-dimensional $C^{*}$-algebras. We always put $\mathrm{A}_{0}=\mathbb{C} 1_{\mathrm{A}}, \mathcal{A}=\bigcup_{n=1}^{\infty} \mathrm{A}_{n}$ and assume that the unit in each $\mathrm{A}_{n}$ is the unit of $A$.

Suppose that $\mathrm{A}$ is acting on a Hilbert space $\mathrm{H}$ and that $\xi \in \mathrm{H}$ is a separating and cyclic unit vector for $\mathrm{A}$. Let $P_{n}$ denote the orthogonal projection onto the subspace $\mathrm{H}_{n}:=\mathrm{A}_{n} \xi$ of $\mathrm{H}$ and write $Q_{0}=P_{0}=P_{\mathbb{C} \xi}, Q_{n}=P_{n}-P_{n-1}$ for $n \in \mathbb{N}$. There exists a (strictly increasing) sequence of real numbers $\left(\alpha_{n}\right)_{n=1}^{\infty}$ such that the selfadjoint operator $D=\sum_{n \in \mathbb{N}} \alpha_{n} Q_{n}$ yields a spectral triple $(\mathcal{A}, \mathrm{H}, D)$ such that the topology on the state space of $A$ induced by the Rieffel metric (Rie] coincides with the weak*-topology. Due to the existence of a cyclic and separating vector the orientation-preserving quantum isometry group exists by Theorem 2.14 of $\mathrm{BG}_{2}$. 
In $\mathrm{CI}_{1}$ it was additionally observed that if $\mathrm{A}$ is infinite-dimensional and $p>0$, then one can choose $\left(\alpha_{n}\right)_{n=1}^{\infty}$ in such a way that the resulting Fredholm module is $p$-summable $\left(\mathrm{Co}_{1}\right)$. This reflects the fact that $A F$ algebras should be thought of as 0 -dimensional noncommutative spaces.

Note that for each $n \in \mathbb{N}$ by restricting we obtain a (finite-dimensional) spectral triple $\left(\mathrm{A}_{n}, \mathrm{H}_{n},\left.D\right|_{\mathrm{H}_{n}}\right)$. As we are precisely in the framework of Theorem [1.2 to compute $\mathrm{QISO}^{+}(\mathcal{A}, \mathrm{H}, D)$ we need to understand the quantum isometry groups QISO $^{+}\left(\mathcal{A}_{n}, \mathrm{H}_{n},\left.D\right|_{\mathrm{H}_{n}}\right)$ and embeddings relating them. To simplify the notation we will write $\mathrm{S}_{n}:=\mathrm{QISO}^{+}\left(\mathrm{A}_{n}, \mathrm{H}_{n},\left.D\right|_{\mathrm{H}_{n}}\right)$.

We begin with some general observations.

Lemma 2.1. Let $\mathrm{QU}_{\mathrm{A}_{n}, \omega_{\xi}}$ denote the universal quantum group acting on $\mathrm{A}_{n}$ and preserving the (faithful) state on $\mathrm{A}_{n}$ given by the vector $\xi$ (see Wan]). There exists a c.q.g. morphism from $\mathrm{QU}_{\mathrm{A}_{n}, \omega_{\xi}}$ to $\mathrm{S}_{n}$.

Proof. The proof is based on considering the spectral triple given by $\left(\mathrm{A}_{n}, \mathrm{H}_{n}, D_{n}^{\prime}\right)$, where $D_{n}^{\prime}=P_{n}-P_{0}$. It is then easy to see that $\mathrm{QISO}^{+}\left(\mathrm{A}_{n}, \mathrm{H}_{n}, D_{n}^{\prime}\right)$ is isomorphic to the universal compact quantum group acting on $A_{n}$ and preserving $\omega_{\xi}$. On the other hand, universality assures the existence of the c.q.g. morphism from $\mathrm{QISO}^{+}\left(\mathrm{A}_{n}, \mathrm{H}_{n}, D_{n}^{\prime}\right)$ to $\mathrm{S}_{n}$.

Lemma 2.2. Assume that each $\mathrm{A}_{n}$ is commutative, $\mathrm{A}_{n}=\mathbb{C}^{k_{n}}, n \in \mathbb{N}$. There exists a c.q.g. morphism from $\mathrm{QU}_{k_{n}}$ to $\mathrm{S}_{k_{n}}$, where $\mathrm{QU}_{k_{n}}$ denotes the universal quantum group acting on $k_{n}$ points (Wan]).

Proof. The proof is identical to the one above. We only need to observe additionally that for any measure $\mu$ on the set $\left\{1, \ldots, k_{n}\right\}$ which has full support there is a natural c.q.g. morphism from $\mathrm{QU}_{k_{n}}$ to $\mathrm{QU}_{\mathbb{C}^{k_{n}, \mu}}$. In the case when $\mu$ is uniformly distributed, we simply have $\mathrm{QU}_{\mathbb{C}^{k_{n}, \mu}}=\mathrm{QU}_{k_{n}}$, as follows from the first part of Lemma 2.4 below.

Let $\alpha_{n}: \mathrm{A}_{n} \rightarrow \mathrm{A}_{n} \otimes \mathrm{S}_{n}$ denote the universal action (on the $n$-th level). Then we have the following important property, being the direct consequence of Theorem 1.2. We have

$$
\alpha_{n+1}\left(\mathrm{~A}_{n}\right) \subset \mathrm{A}_{n} \otimes \mathrm{S}_{n+1}
$$

(where we identified $A_{n}$ with a subalgebra of $A_{n+1}$ ) and $S_{n}$ is generated exactly by these coefficients of $S_{n+1}$ which appear in the image of $\mathrm{A}_{n}$ under $\alpha_{n+1}$. This in conjunction with the previous lemma suggests the strategy for computing relevant quantum isometry groups inductively. Suppose that we have determined the generators of $\mathrm{S}_{n}$. Then $\mathrm{S}_{n+1}$ is generated by generators of $\mathrm{S}_{n}$ and those of the $\mathrm{QU}_{\mathrm{A}_{n}, \omega_{\xi}}$, with the only additional relations provided by the equation (2.1).

This will be used below to determine the concrete form of relations determining $\mathrm{S}_{n}$ for the commutative $A F$ algebras.

Lemma 2.3. Let $\mathrm{A}$ be a commutative AF algebra. Suppose that $\mathrm{A}_{n}$ is isomorphic to $\mathbb{C}^{m}$ and the embedding of $\mathrm{A}_{n}$ into $\mathrm{A}_{n+1}$ is given by a sequence $\left(l_{i}\right)_{i=1}^{m}$. Let $m^{\prime}=$ $\sum_{i=1}^{m} l_{i}$. Suppose that the 'copy' of $\mathrm{QU}_{m}$ in $\mathrm{S}_{n}$ is given by the family of projections $a_{i, j}(i, j \in\{1, \ldots m\})$ and that the 'copy' of $\mathrm{QU}_{m^{\prime}}$ in $\mathrm{S}_{n+1}$ is given by the family of projections $a_{\left(i, r_{i}\right),\left(j, s_{j}\right)}\left(i, j \in\{1, \ldots, m\}, r_{i} \in\left\{1, \ldots, l_{i}\right\}, s_{j} \in\left\{1, \ldots, l_{j}\right\}\right)$. Then 
the formula (2.1) is equivalent to the following system of equalities:

$$
a_{i, j}=\sum_{r_{i}=1}^{l_{i}} a_{\left(i, r_{i}\right),\left(j, s_{j}\right)}
$$

for each $i, j \in\{1, \ldots, m\}, s_{j} \in\left\{1, \ldots, l_{j}\right\}$.

Proof. We have (for the universal action $\alpha: \mathrm{A}_{n} \rightarrow \mathrm{A}_{n} \otimes \mathrm{S}_{n}$ )

$$
\alpha\left(\widetilde{e_{i}}\right)=\sum_{j=1}^{m} \widetilde{e_{j}} \otimes a_{i, j}
$$

where by $\widetilde{e_{i}}$ we denote the image of the basis vector $e_{i} \in \mathrm{A}_{n}$ in $\mathrm{A}_{n+1}$. As $\widetilde{e_{j}}=$ $\sum_{r_{j}=1}^{l_{j}} e_{\left(j, s_{j}\right)}$

$$
\alpha\left(\widetilde{e_{i}}\right)=\sum_{r_{i}=1}^{l_{i}} \alpha\left(e_{i, r_{i}}\right)=\sum_{r_{i}=1}^{l_{i}} \sum_{j=1}^{m} \sum_{s_{j}=1}^{l_{j}} e_{\left(j, s_{j}\right)} \otimes a_{\left(i, r_{i}\right),\left(j, s_{j}\right)} .
$$

On the other hand we have

$$
\alpha\left(\widetilde{e}_{i}\right)=\sum_{j=1}^{m} \sum_{s_{j}=1}^{l_{j}} e_{\left(j, s_{j}\right)} \otimes a_{i, j},
$$

and the comparison of the formulas above yields exactly (2.2).

One can deduce from the above lemma the exact structure of generators and relations between them for each $\mathrm{S}_{n}$ associated with a commutative $A F$ algebra. To be precise, if $\mathrm{A}_{n}=\mathbb{C}^{k_{n}}$ for some $k_{n} \in \mathbb{N}$, then the quantum isometry group $\mathrm{S}_{n}$ is generated as a unital $C^{*}$-algebra by the family of selfadjoint projections $\bigcup_{i=1}^{n}\left\{a_{\alpha_{i}, \beta_{i}}: \alpha_{i}, \beta_{i}=1, \cdots, k_{i}\right\}$ such that for each fixed $i=1, \ldots, n$ the family $\left\{a_{\left(\alpha_{i}, \beta_{i}\right)}: \alpha_{i}, \beta_{i}=1, \cdots, k_{i}\right\}$ satisfies the relations of $\mathrm{QU}_{k_{n}}$ and the additional relations between $a_{\left(\alpha_{i}, \beta_{i}\right)}$ and $a_{\left(\alpha_{i+1}, \beta_{i+1}\right)}$ for $i \in\{1, \ldots, n-1\}$ are given by the formulas (2.2), after suitable reinterpretation of indices according to the multiplicities in the embedding of $\mathbb{C}^{k_{i}}$ into $\mathbb{C}^{k_{i+1}}$.

In $[\mathrm{Bic}$, J. Bichon introduced the notion of a quantum symmetry group of a finite directed graph. As each $A F$ algebra can be described via its Bratteli diagram, it is natural to ask whether the construction in this paper can be compared to the one in Bic. We begin by stating some elementary facts in the following lemma.

Lemma 2.4. Let $\alpha$ be an action of a c.q.g. $\mathrm{S}$ on $C(X)$ where $X$ is a finite set. Then $\alpha$ automatically preserves the functional $\tau$ corresponding to the counting measure:

$$
(\tau \otimes \mathrm{id})(\alpha(f))=\tau(f) \cdot 1_{\mathrm{S}}
$$

Thus $\alpha$ induces a unitary $\widetilde{\alpha} \in \mathcal{B}\left(l^{2}(X)\right) \otimes S$ given by $\widetilde{\alpha}(f \otimes q)=\alpha(f)(1 \otimes q)$. If we define $\alpha^{(2)}: C(X) \otimes C(X) \rightarrow C(X) \otimes C(X) \otimes \mathrm{S}$ by $\alpha^{(2)}=\left(\mathrm{id}_{2} \otimes m_{\mathrm{S}}\right) \sigma_{23}(\alpha \otimes \alpha)$, where $m_{\mathrm{S}}$ denotes the multiplication map from $\mathrm{S} \otimes \mathrm{S}$ to $\mathrm{S}$, and $\mathrm{id}_{2}$ denotes the identity map on $C(X) \otimes C(X)$, then $\alpha^{(2)}$ leaves the diagonal algebra $C\left(D_{X \times X}\right)$ invariant (here $D_{X \times X}=\{(x, x): x \in X\}$ ).

Proof. Let $X=\{1, \ldots, n\}$ for some $n \in \mathbb{N}$ and denote by $\delta_{i}$ the characteristic function of the point $i$. Let $\alpha\left(\delta_{i}\right)=\sum_{j} \delta_{j} \otimes q_{i j}$, where $\left\{q_{i j}: i, j=1 \ldots n\right\}$ are the images of the canonical generators of the quantum permutation group as in 
Wan. Then $\tau$-preservation of $\alpha$ follows from the properties of the generators of the quantum permutation group, which in particular imply that $\sum_{j} q_{i j}=1=\sum_{i} q_{i j}$.

Using the fact that $q_{i j}$ and $q_{i k}$ are orthogonal for $i, j, k \in\{1, \ldots, n\}, j \neq k$, we obtain

$$
\alpha^{(2)}\left(\delta_{i} \otimes \delta_{i}\right)=\sum_{j, k} \delta_{j} \otimes \delta_{k} \otimes q_{i j} q_{i k}=\sum_{j} \delta_{j} \otimes \delta_{j} \otimes q_{i j},
$$

from which the invariance of the diagonal under $\alpha^{(2)}$ is immediate.

The other statements follow easily.

Observe that since $\widetilde{\alpha^{(2)}}$ is a unitary, it leaves the $\tau$-orthogonal complement of $C\left(D_{X \times X}\right)$ in $l^{2}(X \times X)$, i.e., the space of functions on the set $Y=\{(x, y): x, y \in$ $X, x \neq y\}$, invariant as well.

Recall now the definition of the quantum automorphism group of a finite graph given in $[\mathrm{Bic}$. Let $(V, E)$ be a graph with $V$ denoting the set of vertices and $E$ the set of edges. Let $s: E \rightarrow V$ (respectively $t: E \rightarrow V$ ) be the source map (respectively the target map). The target and source maps induce ${ }^{*}$-homomorphisms $s^{*}, t^{*}$ : $C(V) \rightarrow C(E)$. Let $m: C(E) \otimes C(E) \rightarrow C(E)$ be the pointwise multiplication map on $E$ and given a quantum group action $\alpha$ on $C(V)$ let $\alpha^{(2)}$ be defined as in Lemma 2.4 .

Definition $2.5(\overline{\mathrm{Bic}})$. An action of a c.q.g. S on a finite graph $G=(V, E)$ consists of an action $\alpha$ of $\mathrm{S}$ on the set of vertices, $\alpha: C(V) \rightarrow C(V) \otimes \mathrm{S}$ and an action $\beta$ of $\mathrm{S}$ on the set of edges, $\beta: C(E) \rightarrow C(E) \otimes \mathrm{S}$, such that

$$
\left(\left(m\left(s_{*} \otimes t_{*}\right)\right) \otimes \mathrm{id}_{\mathrm{S}}\right) \circ \alpha^{(2)}=\beta \circ\left(m\left(s^{*} \otimes t^{*}\right)\right) .
$$

It is also called a quantum symmetry of the graph $(V, E)$.

The quantum automorphism group of the finite graph is the universal object in the category of compact quantum groups with actions as above. We refer to Bic for the details.

Let us now restrict our attention to a truncation of a Bratteli diagram (up to the $n$-th level, say) of a commutative $A F$ algebra. The set of vertices $V$ is a disjoint union of sets $V_{1}, \ldots, V_{n}$ with $V_{1}$ being a singleton, and there exist surjective maps $\pi_{j}: V_{j} \rightarrow V_{j-1}(j \geq 2)$ determining the graph structure. Denote by $\pi$ the map from $V$ to $V$ defined by the formulas $\left.\pi\right|_{V_{j}}=\pi_{j}$ for $j \geq 2$, and $\pi=$ id on $V_{1}$. Then $\pi^{*}: C(V) \rightarrow C(V)$ is a $C^{*}$-homomorphism, with $\left.\pi^{*}\right|_{C\left(V_{j}\right)}$ injective for each $j \leq n-1$. The corresponding graph is obtained by joining $\pi_{i+1}(v) \in V_{i}$ with $v \in V_{i+1}$ for each $i=1, \ldots, n, v \in V_{i}$.

Denote by $D(V)$ the diagonal subalgebra of $C(V) \otimes C(V)$, i.e. the span of $\left\{\delta_{v} \otimes \delta_{v}\right.$ : $v \in V\}$. Since the map $m\left(s_{*} \otimes t_{*}\right)$ is onto, $C(E) \cong(C(V) \otimes C(V)) / \operatorname{Ker}\left(m\left(s_{*} \otimes t_{*}\right)\right)$. Indeed, for the graph corresponding to the commutative $A F$ algebra described above, $C(E)$ is isomorphic to the subalgebra $\left(\mathrm{id} \otimes \pi^{*}\right)(D(V))$ of $C(V) \otimes C(V)$, and the condition on $\beta$ in the above definition of a quantum symmetry of the graph $(V, E)$ amounts to saying that $\alpha^{(2)}$ leaves the algebra $\mathcal{C}:=\left(\operatorname{id} \otimes \pi^{*}\right)(D(V))$ invariant, in which case $\beta$ is, up to the obvious identification, nothing but the restriction of $\alpha^{(2)}$ on $\mathcal{C}$.

In other words, an equivalent description of the objects in the category of the quantum symmetry of such a finite commutative (i.e. with all matrix algebras in the vertices being one-dimensional) Bratteli diagram is obtained via observing that 
they correspond precisely to these c.q.g. actions $\alpha$ on $C(V)$ for which $\mathcal{C}$ is left invariant by $\alpha^{(2)}$. This leads to the following result:

Theorem 2.6. Let A be a commutative AF algebra. Then the quantum isometry group $\mathrm{S}_{n}$ described in the beginning of this section coincides with the quantum symmetry group of the graph given by the restriction of the Bratteli diagram of $\mathrm{A}$ to the $n$-th level.

Proof. Suppose first that we are given a quantum isometry of the canonical spectral triple on the respective 'finite' part of the $A F$ algebra in question, so that we have a c.q.g. action $(\mathrm{S}, \alpha)$ on $C(V)$ such that each $\left.\alpha_{j} \equiv \alpha\right|_{C\left(V_{j}\right)}$ leaves $C\left(V_{j}\right)$ invariant $(j=1, \ldots, n)$ and that $\alpha$ commutes with the embeddings $\pi_{j}$, that is,

$$
\alpha_{j+1} \pi_{j+1}^{*}=\left(\pi_{j+1}^{*} \otimes \mathrm{id}\right) \alpha_{j} .
$$

We deduce that

$$
\begin{aligned}
\alpha_{j+1}^{(2)}\left(\mathrm{id} \otimes \pi_{j+1}{ }^{*}\right) & =\left(\mathrm{id}_{2} \otimes m_{\mathrm{S}}\right) \sigma_{23}\left(\alpha_{j+1} \otimes \alpha_{j+1} \pi_{j+1}{ }^{*}\right) \\
& =\left(i d_{2} \otimes m_{\mathrm{S}}\right) \sigma_{23}\left(\alpha_{j+1} \otimes\left(\pi_{j+1}{ }^{*} \otimes \mathrm{id}\right) \alpha_{j}\right)=\left(\pi_{j+1}{ }^{*} \otimes \mathrm{id}\right) \alpha_{j}^{(2)} .
\end{aligned}
$$

Using the above expression and the fact that $\alpha^{(2)}$ leaves $D(V)$ invariant (by the second part of Lemma 2.4), we see that $\alpha^{(2)}$ leaves $\mathcal{C}$ invariant.

Conversely, we need to show that a quantum action of a c.q.g. on the Bratteli diagram induces a quantum symmetry of the corresponding part of a spectral triple on the $A F$ algebra. Let $(\mathrm{S}, \alpha)$ be then an action on $C(V)$ such that $\alpha^{(2)}$ leaves $\mathcal{C}$ invariant. It follows from the discussion before the lemma that we have the corresponding action $\beta$ on $C(E)$. Therefore we can start with an action $\alpha$ on the Bratteli diagram such that $\alpha^{(2)} C\left(V_{n}\right)$ preserves (id $\left.\otimes \pi_{n}{ }^{*}\right)\left(D\left(V_{n-1}\right)\right)$. We first show by induction that $\alpha$ leaves each $C\left(V_{j}\right)$ invariant. Consider $j=n$ first. Observe that $C\left(V_{n}\right)$ is nothing but $\operatorname{Ker}(\Psi)$, where $\Psi: C(V) \rightarrow C(E)$ is the map $f \mapsto$ $\left(m \circ\left(s^{*} \otimes t^{*}\right)\right)(f \otimes 1)$. It is clear from the definition of a quantum symmetry of a graph that $\alpha$ will leave this subalgebra invariant. This implies that $\alpha$ (being a unitary w.r.t. the counting measure on $C(V))$ will leave $C\left(V_{1} \cup \ldots \cup V_{n-1}\right)$ invariant as well, and thus restricts to a quantum symmetry of the reduced graph obtained by deleting $V_{n}$ and the corresponding edges. Then the inductive arguments complete the proof that each $C\left(V_{j}\right)$ is left invariant.

The proof will now be complete if we can show that

$$
\alpha_{m+1} \pi_{m+1}^{*}=\left(\pi_{m+1}^{*} \otimes \mathrm{id}\right) \alpha_{m}
$$

for each $m=1, \ldots, n-1$. Let $V_{m}=\left\{v_{1}^{m}, \ldots, v_{t_{m}}^{m}\right\}$, and let $q_{m, l j}$ be elements of $\mathrm{S}$ such that

$$
\alpha\left(\delta_{v_{l}^{m}}\right)=\sum_{j} \delta_{v_{j}^{m}} \otimes q_{m, l j} .
$$

We set $\Lambda_{j}=\{m: \pi(m)=j\}$. Then we have

$$
\begin{aligned}
\alpha^{(2)}\left(\mathrm{id} \otimes \pi_{m+1}^{*}\right)\left(\delta_{v_{i}^{m}} \otimes \delta_{v_{i}^{m}}\right) & =\alpha^{(2)}\left(\delta_{v_{i}^{m}} \otimes \sum_{k \in \Lambda_{i}} \delta_{v_{k}^{m+1}}\right) \\
& =\sum_{j, p} \sum_{k \in \Lambda_{i}} \delta_{v_{j}^{m}} \otimes \delta_{v_{p}^{m+1}} \otimes q_{m, i j} q_{m+1, k p} .
\end{aligned}
$$


Further then for all $p \notin \Lambda_{j}$,

$$
\sum_{k \in \Lambda_{i}} q_{m, i j} q_{m+1, k p}=0
$$

Multiplying by $q_{m+1, k^{\prime} p}$, where $k^{\prime} \in \Lambda_{i}$, we obtain that

$$
\forall_{p \notin \Lambda_{j}, k \in \Lambda_{i}} \quad q_{m, i j} q_{m+1, k p}=0 .
$$

As stated after Lemma 2.4, $\alpha^{(2)}$ leaves the ortho-complement of the diagonal algebra in $l^{2}(V \times V)$ invariant. This means that if $k \notin \Lambda_{i}$, then $\alpha^{(2)}\left(\delta_{v_{i}^{m}} \otimes \delta_{v_{k}^{m+1}}\right)$ belongs to $C\left(D^{c}\right) \otimes \mathrm{S}$. On the other hand,

$$
\alpha^{(2)}\left(\delta_{v_{i}^{m}} \otimes \delta_{v_{k}^{m+1}}\right)=\sum_{j, p} \delta_{v_{j}^{m}} \otimes \delta_{v_{p}^{m+1}} \otimes q_{m, i j} q_{m+1, k p},
$$

So

$$
\forall_{k \notin \Lambda_{i}, p \in \Lambda_{j}} \quad q_{m, i j} q_{m+1, k p}=0 .
$$

Further

$$
\alpha_{m+1} \pi_{m+1}^{*}\left(\delta_{v_{i}^{m}}\right)=\sum_{j} \delta_{v_{j}^{m+1}} \otimes \sum_{k \in \Lambda_{i}} q_{m+1, k j}
$$

We also have

$$
\left(\pi_{m+1}^{*} \otimes \mathrm{id}\right) \alpha_{m}\left(\delta_{v_{i}^{m}}\right)=\sum_{j} \delta_{v_{j}^{m+1}} \otimes \sum_{r: j \in \Lambda_{r}} q_{m, i r}=\sum_{j} \delta_{v_{j}^{m+1}} \otimes q_{m, i \pi(j)} .
$$

Finally

$$
\begin{aligned}
\sum_{k \in \Lambda_{i}} q_{m+1, k p} & =\sum_{k \in \Lambda_{i}}\left(\sum_{j} q_{m, i j}\right) q_{m+1, k p}=\sum_{k \in \Lambda_{i}}\left(\sum_{j} q_{m, i j} q_{m+1, k p}\right) \\
& =\sum_{k \in \Lambda_{i}} q_{m, i \pi(p)} q_{m+1, k p}=\sum_{k} q_{m, i \pi(p)} q_{m+1, k p}-\sum_{k \notin \Lambda_{i}} q_{m, i \pi(p)} q_{m+1, k p} \\
& =q_{m, i \pi(p)},
\end{aligned}
$$

where in the third equality we used (2.4) and in the final equality we used (2.5) as well as the relation $\sum_{k} q_{m+1, k p}=1$. This shows that (2.3) holds and the proof is finished.

The above result justifies the statement that the quantum isometry groups of Christensen-Ivan triples on $A F$ algebras provide natural notions of quantum symmetry groups of the corresponding Bratteli diagrams. The theorem could be proved directly by comparing the commutation relations of [Bic] with those listed in Lemma 2.3. but the method we gave has the advantage of being more functorial and transparent.

\section{The EXAmple For the Middle-third Cantor Set}

In the special case when $\mathrm{A}$ is the (commutative) $A F$ algebra of continuous functions on the middle-third Cantor set we can use the observations of the last section to provide an explicit description of the quantum isometry groups $S_{n}$, and therefore also of $\widetilde{\mathrm{QISO}}^{+}(\mathcal{A}, \mathrm{H}, D)$. Note that several variants of the spectral triple we consider here were studied in $\mathrm{CI}_{1}$ and in $\mathbf{C I}_{2}$, where its construction is attributed to the unpublished work of Connes. 
Theorem 3.1. Let $\mathrm{A}$ be the AF algebra arising as a limit of the unital embeddings

$$
\mathbb{C}^{2} \longrightarrow \mathbb{C}^{2} \otimes \mathbb{C}^{2} \longrightarrow \mathbb{C}^{2} \otimes \mathbb{C}^{2} \otimes \mathbb{C}^{2} \longrightarrow \cdots
$$

Suppose that the state $\omega_{\xi}$ is the canonical trace on $\mathrm{A}$. Then $\mathrm{S}_{1}=C\left(\mathbb{Z}_{2}\right)$ and for $n \in \mathbb{N}$,

$$
\mathrm{S}_{n+1}=\left(\mathrm{S}_{n} \star \mathrm{S}_{n}\right) \oplus\left(\mathrm{S}_{n} \star \mathrm{S}_{n}\right) .
$$

Proof. Begin by noticing that for each $n \in \mathbb{N}$ we have $\mathrm{A}_{n}=\mathbb{C}^{2^{n}}$ and therefore we can use a natural multi-index notation for the indexing sets discussed in the paragraph after Lemma 2.3. Precisely speaking, denote for each $n \in \mathbb{N}$ by $\mathcal{J}_{n}$ the set $\left\{i_{1} i_{2} \cdots i_{n}: i_{j} \in\{1,2\}\right.$ for $\left.j=1, \ldots, n\right\}$. Multi-indices in $\mathcal{J}:=\bigcup_{n \in \mathbb{N}} \mathcal{J}_{n}$ will be denoted by capital letters $I, J, \ldots$, and let the basis of the algebra $\mathrm{A}_{n}$ be indexed by elements of $\mathcal{J}_{n}$. Then the natural embeddings between $A_{n}$ and $A_{n+1}$ can be conveniently described by the formula

$$
e_{I} \longrightarrow e_{I 1}+e_{I 2}, \quad I \in \mathcal{J}_{n},
$$

where we use the standard concatenation of multi-indices. The equations (2.2) now take the following form:

$$
a_{I, J}=a_{I 1, J 1}+a_{I 2, J 1}=a_{I 1, J 2}+a_{I 2, J 2}, \quad I, J \in \mathcal{J} .
$$

Note that so far the fact that at every step we 'divided the set' into 2 parts did not play any significant role; we could adopt the multi-index notation replacing 2 by $3,4, \ldots$. Now analyse the equations (3.1) remembering that both $\left\{a_{I, J}: I, J \in \mathcal{J}_{n}\right\}$ and $\left\{a_{I^{\prime}, J^{\prime}}: I^{\prime}, J^{\prime} \in \mathcal{J}_{n+1}\right\}$ form 'magic unitaries' whose entries are orthogonal projections ([Wan $)$. Fix $I \in \mathcal{J}_{n}$ and consider the respective equalities

$$
1=\sum_{J \in \mathcal{J}_{n}} a_{I, J}=\sum_{J \in \mathcal{J}_{n}}\left(a_{I 1, J 1}+a_{I 2, J 1}\right)
$$

and

$$
1=\sum_{J^{\prime} \in \mathcal{J}_{n+1}} a_{I 1, J^{\prime}}=\sum_{J \in \mathcal{J}_{n}}\left(a_{I 1, J 1}+a_{I 1, J 2}\right) .
$$

They imply that

$$
\sum_{J \in \mathcal{J}_{n}} a_{I 2, J 1}=\sum_{J \in \mathcal{J}_{n}} a_{I 1, J 2} .
$$

By formulas (3.1), each of the respective factors in the sum above is an orthogonal subprojection of the projection $a_{I, J}$, and the projections $a_{I, J}, a_{I, K}$ are mutually orthogonal when $J \neq K$. Therefore we actually must have

$$
a_{I 2, J 1}=a_{I 1, J 2}
$$

for each $J \in \mathcal{J}_{n}$. Using once again (3.1) we see that actually

$$
a_{I 1, J 1}=a_{I 2, J 2}=a_{I, J}-a_{I 1, J 2}=a_{I, J}-a_{I 2, J 1}, \quad I, J \in \mathcal{J}_{n} .
$$

This means that the choice of $a_{I 1, J 1}$ determines already the remaining three projections. Observe also that if we fix the family $\left\{a_{I, J}: I, J \in \mathcal{J}_{n}\right\}$ which forms a magic unitary whose entries are selfadjoint projections, choose for each $I, J \in \mathcal{J}_{n}$ a subprojection $a_{I 1, J 1}$ of $a_{I, J}$ and define the projections $a_{I 2, J 1}, a_{I 1, J 2}, a_{I 2, J 2}$ by formulas (3.2), then the resulting family $\left\{a_{I^{\prime}, J^{\prime}}: I^{\prime}, J^{\prime} \in \mathcal{J}_{n+1}\right\}$ automatically yields a magic unitary whose entries are orthogonal projections.

Let us now see what the above discussion tells us about the structure of $S_{n}$ in this particular case. Start with $A_{1}$. As $A_{0}=\mathbb{C}$, the invariance condition on the 
embedding means simply that the action is unital and we see that $\mathrm{S}_{1}$ is simply the universal quantum group acting on 2 points, $C\left(\mathbb{Z}_{2}\right)$. Denote $a_{11}=p$. Then the unitary matrix corresponding to $\left\{a_{I, J}: I, J \in \mathcal{J}_{1}\right\}$ looks as follows:

$$
\left(\begin{array}{cc}
p & p^{\perp} \\
p^{\perp} & p
\end{array}\right)
$$

In the second step, according to formulas (3.2), the 4 by 4 magic unitary looks as follows:

$$
\left(\begin{array}{cccc}
q_{1} & p-q_{1} & q_{2} & p^{\perp}-q_{2} \\
p-q_{1} & q_{1} & p^{\perp}-q_{2} & q_{2} \\
q_{3} & p^{\perp}-q_{3} & q_{4} & p-q_{4} \\
p^{\perp}-q_{3} & q_{3} & p-q_{4} & q_{4}
\end{array}\right),
$$

where $q_{1}, q_{3}$ are orthogonal subprojections of $p$ and $q_{2}, q_{4}$ are orthogonal subprojections of $p^{\perp}$. The quantum group $\mathrm{S}_{2}$ is then generated by the projections $p, q_{1}, q_{2}, q_{3}, q_{4}$ subjected to constraints described in the previous sentence (note that the subordination for projections can be formulated in terms of the usual algebraic relations: $q \leq q^{\prime}$ if and only if $q q^{\prime}=0$ ). The $C^{*}$-algebraic structure of $\mathrm{S}_{2}$ can be seen as follows: the projection $p$ provides a decomposition of $\mathrm{S}_{2}$ into a direct sum, and then in each of the factors we choose independently two orthogonal projections (respectively $q_{1}$ and $q_{3}$ or $q_{2}$ and $q_{4}$ ). As there are no relations between $q_{1}$ and $q_{3}$, the universal $C^{*}$-algebra they generate is simply $C\left(\mathbb{Z}_{2}\right) \star C\left(\mathbb{Z}_{2}\right)$, the universal algebra generated by two orthogonal projections (see Cha ). Thus

$$
\mathrm{S}_{2}=\left(C\left(\mathbb{Z}_{2}\right) \star C\left(\mathbb{Z}_{2}\right)\right) \oplus\left(C\left(\mathbb{Z}_{2}\right) \star C\left(\mathbb{Z}_{2}\right)\right) .
$$

The Hopf*- algebraic structure can be read immediately from the condition that the matrix (3.3) gives a corepresentation of $S_{2}$. The inductive reasoning should now be clear. It can be visualised by the sequence of pictures, representing consecutive subdivisions of a square. The fractal structure of the limiting algebra is apparent. Note also that the classical symmetry group of the tree-type graph we consider can be graphically interpreted as a one-dimensional version of the above two-dimensional picture (so that the classical symmetry group $\mathbf{S}_{n}^{\text {clas }}$ is simply equal to $\left.\prod_{i=1}^{2^{n}} \mathbb{Z}_{2}\right)$.

We can also give a description of the $\lim _{n \in \mathbb{N}} S_{n}$ in terms of the generators and relations:

Corollary 3.2. The quantum isometry group of a natural spectral triple on the algebra of continuous functions on the middle-third Cantor set constructed in $\mathbf{C I}_{1}$ is the universal $C^{*}$-algebra generated by the family of selfadjoint projections

$$
\{p\} \cup \bigcup_{n \in \mathbb{N}}\left\{p_{m_{1}, \ldots m_{n}}: m_{1}, \ldots, m_{n} \in\{1,2,3,4\}\right\}
$$

subjected to the following relations:

$$
\begin{gathered}
p_{1}, p_{2} \leq p, \quad p_{3}, p_{4} \leq p^{\perp}, \\
p_{m_{1}, \ldots, m_{n}, 1}, p_{m_{1}, \ldots, m_{n}, 2} \leq p_{m_{1}, \ldots, m_{n}}, \quad p_{m_{1}, \ldots, m_{n}, 3}, p_{m_{1}, \ldots, m_{n}, 4} \leq p_{m_{1}, \ldots, m_{n}}^{\perp} \\
\left(n \in \mathbb{N}, m_{1}, \ldots, m_{n} \in\{1,2,3,4\}\right) .
\end{gathered}
$$

Proof. A straightforward consequence of Theorem 1.2 and Lemma 3.1 . 
It is clear from the proof of Theorem 3.1 and also from the discussion before Lemma 2.3 that the quantum group actions we consider are actions on the tensor product of algebras preserving in some sense one of the factors. In the classical world such actions have to have a product form, as the following lemma confirms:

Lemma 3.3. Suppose that $\left(X, d_{X}\right),\left(Y, d_{Y}\right)$ are compact metric spaces and $T$ : $X \times Y \rightarrow X \times Y$ is an isometry satisfying the following condition: $\alpha_{T}\left(C(X) \otimes 1_{Y}\right) \subset$ $C(X) \otimes 1_{Y}$, where $\alpha_{T}: C(X \times Y) \rightarrow C(X \times Y)$ is given simply by the composition with $T$. Then $T$ has to be a product isometry.

Proof. Denote the family of isometries of $X \times Y$ satisfying the conditions of the lemma by $\mathrm{ISO}_{X}(X \times Y)$. We claim that $\mathrm{ISO}_{X}(X \times Y)$ is a group. Recall that $\operatorname{ISO}(Z)$, the family of all isometries of a compact metric space $\left(Z, d_{Z}\right)$, is a compact group when considered with the topology of uniform convergence (equivalently, pointwise convergence; equivalently, metric topology given by $d\left(T_{1}, T_{2}\right)=$ $\sum_{i=1}^{\infty} \frac{1}{2^{i}} d_{Z}\left(T_{1}\left(z_{i}\right), T_{2}\left(z_{i}\right)\right)$, where $\left\{z_{i}: i \in \mathbb{N}\right\}$ is a countable dense subset of $\left.Z\right)$. It is easy to show that ISO $_{X}(X \times Y)$ is a unital closed subsemigroup of $\operatorname{ISO}(X \times Y)$. Thus it is a compact semigroup satisfying the cancellation properties and it has to be closed under taking inverses.

Suppose now that $T \in \mathrm{ISO}_{X}(X \times Y)$. Then if $f \in C(X)$ we have for all $x \in$ $X, y, y^{\prime} \in Y$,

$$
\left(f \otimes 1_{Y}\right)(T(x, y))=\alpha_{T}\left(f \otimes 1_{Y}\right)(x, y)=\alpha_{T}\left(f \otimes 1_{Y}\right)\left(x, y^{\prime}\right)=\left(f \otimes 1_{Y}\right)\left(T\left(x, y^{\prime}\right)\right) .
$$

This is equivalent to the fact that $T$ is given by the formula

$$
T(x, y)=(h(x), g(x, y)), \quad x \in X, y \in Y,
$$

for some transformations $h: X \rightarrow X, g: X \times Y \rightarrow Y$. The fact that $T$ is an isometry implies in particular that for all $x, x^{\prime} \in X, y \in Y$,

$$
d_{X}\left(x, x^{\prime}\right)=d_{X}\left(h(x), h\left(x^{\prime}\right)\right)+d_{Y}\left(g(x, y), g\left(x^{\prime}, y\right)\right) .
$$

In particular, $h: X \rightarrow X$ is a contractive transformation. As by the first part of the proof $T^{-1} \in \mathrm{ISO}_{X}(X \times Y)$, there exist transformations $h^{\prime}: X \rightarrow X, g^{\prime}: X \times Y \rightarrow Y$ such that

$$
T^{-1}(x, y)=\left(h^{\prime}(x), g^{\prime}(x, y)\right), \quad x \in X, y \in Y .
$$

It is easy to see that $h^{\prime}$ is the inverse transformation of $h$, and as by the same argument as above we see that $h^{\prime}$ is a contractive transformation, hence $h$ has to be an isometry. This together with formula (3.4) implies that $g: X \times Y \rightarrow Y$ does not depend on the first coordinate, so that $T$ must be a product isometry. In particular $\mathrm{ISO}_{X}(X \times Y)=\mathrm{ISO}_{X} \times \mathrm{ISO}_{Y}$.

Theorem 3.1 shows that the result above has no counterpart for quantum group actions, even on classical spaces. We could have thought of elements of $S_{2}$ as quantum isometries acting on the Cartesian product of 2 two-point sets, 'preserving' the first coordinate in the sense analogous to the one in the lemma above. If this forced elements of $S_{2}$ to be product isometries, we would necessarily have $\mathrm{S}_{2}=\mathrm{S}_{1} \otimes \mathrm{S}_{1}$; in particular, $\mathrm{S}_{2}$ would have to be commutative. 


\section{QUANTUM SYMMETRY GROUPS OF FINITE METRIC SPACES AND FINITE GRAPHS AS QUANTUM ISOMETRY GROUPS OF CERTAIN NATURAL SPECTRAL TRIPLES}

Theorem 2.6 shows that the quantum symmetry group of a particular type of a finite graph (as defined in $[\mathrm{Bic}$ ) coincides with the quantum isometry group of a certain spectral triple. Motivated by this we show in this section that given a finite metric space $X$ the quantum symmetry group of $X$ defined in Ban coincides with the quantum isometry group of the algebra of functions on $X$ equipped with the natural Laplacian. We also discuss the connections with the natural Dirac operator on a particular representation of $C(X)$, for which the Rieffel-type metric (Rie]) on the state space of $C(X)$ restricts to the original metric on $X$. It can be seen, as pointed out earlier in $\mathrm{Ban}$, that this framework can be related to the one of quantum symmetry groups of finite graphs.

Let $(X, d)$ be a finite metric space of $n$ points. For simplicity we will write $X=\{1, \ldots, n\}$ and for $i, j \in X$ define $d_{i j}=d(i, j)$. As in Section 2 we will denote by $\delta_{i}$ the indicator function of the point $i \in X$ and by $D_{X \times X}$ the diagonal in $X \times X$.

The metric structure on $X$ allows the construction of a natural spectral triple on $C(X)$ (see $\mathrm{Rie}, \mathrm{CI}_{2}$ ). Let $Y=X \times X-D_{X \times X}$, define the Hilbert space $\mathrm{H}=\bigoplus_{\left(x_{0}, x_{1}\right), x_{0} \neq x_{1}} \mathrm{H}_{\left(x_{0}, x_{1}\right)}$, where $\mathrm{H}_{\left(x_{0}, x_{1}\right)}=\mathbb{C}^{2}$, and let the Dirac operator be given by

$$
D=\bigoplus_{\left(x_{0}, x_{1}\right), x_{0} \neq x_{1}} d^{-1}\left(x_{0}, x_{1}\right)\left(\begin{array}{cc}
0 & i \\
-i & 0
\end{array}\right)
$$

When we view $\mathrm{H}$ as $\mathbb{C}^{2} \otimes l^{2}(Y)$, then

$$
D=\left(\begin{array}{cc}
0 & i \\
-i & 0
\end{array}\right) \otimes M_{d^{-1}}
$$

where $M_{d^{-1}}$ denotes multiplication by the function $d^{-1}$ on $Y$.

Let $s, t: Y \rightarrow X$ be given by the formulas $s\left(x_{0}, x_{1}\right)=x_{0}, t\left(x_{0}, x_{1}\right)=x_{1}$. Then for each $f \in C(X)$ there is $s^{*}(f)=(f \otimes 1) \chi_{Y}, t^{*}(f)=(1 \otimes f) \chi_{Y}$, where $\chi_{Y}$ denotes the characteristic function of $Y$. For $f \in C(X), j=1,2$, let

$$
\pi_{1}^{(j)}(f)=\left(\begin{array}{ll}
1 & 0 \\
0 & 0
\end{array}\right) \otimes s^{*}(f), \quad \pi_{2}^{(j)}(f)=\left(\begin{array}{cc}
0 & 0 \\
0 & 2-j
\end{array}\right) \otimes t^{*}(f),
$$

define $\pi_{2}^{(j)}(f)=\pi_{1}^{(j)}(f)+\pi_{2}^{(j)}(f)$ and consider the spectral triple $\left(C(X), \mathrm{H}, \pi^{(j)}, D\right)$. Denote by $\mathbf{C}_{\mathbf{X}, \mathbf{d}}^{(\mathbf{j})}$ the category of compact quantum groups acting by volume (corresponding to $R=I$ ) and orientation-preserving isometries on the above spectral triple. By Theorem 2.10 in $\mathrm{BG}_{2}$, the universal object in this category exists; we denote it by $\widetilde{\mathrm{QISO}}_{I}^{+}(D)$.

It is also easy to describe the 'Laplacian' in the sense of Gos for the spectral triples.

Lemma 4.1. The Laplacian $\mathcal{L}$ on $C(X)$ associated with the spectral triple constructed above via the prescription in [Gos] for $\left(C(X), \mathrm{H}, \pi^{(1)}, D\right)$ is given by

$$
\mathcal{L}\left(\delta_{i}\right)=\frac{4}{2 n-1} \sum_{j \in X} c(i, j) \delta_{j}, \quad \text { i in } X,
$$

where for $i, j \in X$ we have $c(i, j)=d^{-2}(i, j)$ if $i \neq j$ and $c(i, i)=0$. 
Proof. Let $\tau_{0}$ be the functional on $C(X)$ defined by $\tau_{0}(f)=\sum_{i=1}^{n} f(i)$. Denote by $\mathrm{H}_{0}$ the Hilbert space obtained by completing $\pi(C(X))$ with respect to the norm coming from the functional $\operatorname{Tr}(\pi(f))$. It is easy to see that $\operatorname{Tr}(\pi(f))=(2 n-1) \tau_{0}(f)$.

From the definition of the inner product it follows that for all $f, g \in C(X)$,

$$
\langle\mathcal{L}(f), g\rangle=\sum_{i \in X} g(i)(2 n-1) \overline{\mathcal{L}(f)(i)}
$$

On the other hand, $\langle\mathcal{L}(f), g\rangle=-\left\langle d_{D}^{*} d_{D} f, g\right\rangle=-\left\langle d_{D} f, d_{D} g\right\rangle$ (where $d_{D}(\cdot)=$ $[D, \cdot]$ as in Gos $)$, which by a routine calculation can be shown to be equal to $4 \sum_{i \neq j} \frac{g(i)(\bar{f}(j)-\bar{f}(i)}{d^{2}(i, j)}$. Thus for each $i \in X$,

$$
\mathcal{L}(f)(i)=\frac{4}{2 n-1} \sum_{j \neq i} \frac{f(j)-f(i)}{d^{2}(i, j)} .
$$

Comparison of the above with formula (4.1) ends the proof.

It is now easy to verify that $\mathcal{L}$ is admissible in the sense of Gos, so that the corresponding quantum isometry group $\operatorname{QISO}^{\mathcal{L}}(X)$ exists. Recall that $\operatorname{QISO}^{\mathcal{L}}(X)$ is the universal object in the category $\mathbf{C}_{\mathbf{X}, \mathbf{d}}^{\mathcal{L}}$, with the objects being pairs $(\mathrm{S}, \alpha)$, where $\mathrm{S}$ is a compact quantum group and $\alpha$ is the action of $\mathrm{S}$ on $C(X)$ satisfying $(\mathcal{L} \otimes$ id $) \circ \alpha=\alpha \circ \mathcal{L}$. We want to compare $\operatorname{QISO}^{\mathcal{L}}(X)$ with the universal quantum symmetry group of $X$ constructed by Banica in $B$ an. To this end we first need to observe the alternative characterisation of the actions of compact quantum groups on finite metric spaces considered in $\mathrm{Ban}$.

Lemma 4.2. Given an action $\alpha$ of a compact quantum group $\mathrm{S}$ on a finite metric space $(X, d)$ (i.e., an action of $\mathrm{S}$ on $C(X)$ ), the following are equivalent:

(i) $(\mathrm{S}, \alpha)$ is a quantum isometry in the sense of Banica ([Ban $)$;

(ii) $\alpha^{(2)}(d)=d \otimes 1$;

(iii) $\alpha^{(2)}(c)=c \otimes 1$, where $c \in C(X \times X)$ is as in Lemma 4.1;

(iv) $(\mathrm{S}, \alpha)$ is an object in $\mathbf{C}_{\mathbf{X}, \mathbf{d}}^{\mathcal{L}}$.

Proof. (i) $\Leftrightarrow$ (ii): Write $d=\sum_{i, j \in X} d_{i j} \delta_{i} \otimes \delta_{j}$, where $d_{i j}$ are defined as in the beginning of this section. Let us write the action $\alpha$ as

$$
\alpha\left(\delta_{i}\right)=\sum_{j \in X} \delta_{j} \otimes q_{i j},
$$

where $q_{i j} \in \mathrm{S}$. Then it follows by using the relations of the quantum permutation group that the relation $\alpha^{(2)}(d)=d \otimes 1$ is equivalent to the following equation being satisfied for all $k, l \in X$ :

$$
d_{k l} 1=\sum_{i, j \in X} d_{i j} q_{k i} q_{l j}
$$

Thus, to prove the lemma, it is enough to show equivalence of (4.3) with Banica's definition of quantum isometry.

Begin by noting that Banica's definition implies that for all $k, l \in X$,

$$
\sum_{i \in X} d_{i l} q_{k i}=\sum_{i \in X} d_{k i} q_{i l}
$$


From this, it follows that for all $k, m \in X$,

$$
\sum_{l, i \in X} d_{i l} q_{k i} q_{m l}=\sum_{l, i \in X} d_{k i} q_{i l} q_{m l}=\sum_{l \in X} d_{k m} q_{m l}=d_{k m} 1,
$$

which is exactly (4.3).

For the converse direction rewrite (4.3) as

$$
\begin{aligned}
\sum_{i \in X} d_{k i} q_{i l} & =\sum_{i \in X}\left(\sum_{j, m \in X} d_{j m} q_{k j} q_{i m}\right) q_{i l}=\sum_{i, j, m \in X} d_{j m} q_{k j} q_{i m} q_{i l} \\
& =\sum_{i, j, l \in X} q_{k j} \delta_{m, l} d_{j m} q_{i m} q_{i l} \text { (where } \delta_{m, l} \text { denotes the Kronecker delta) } \\
& =\sum_{i, j \in X} q_{k j} d_{j l} q_{i l}=\sum_{j \in X} d_{j l} q_{k j} .
\end{aligned}
$$

Thus Banica's condition is derived.

The proof of (iii) $\Leftrightarrow$ (iv) is very similar to the above proof of equivalence of (i) and (ii), hence is omitted.

Finally, the equivalence of (ii) and (iii) follows from the relation between $c$ and $d$, i.e.

$$
c=\chi_{Y} d^{-2}, d=\left(1-\chi_{Y}+c\right)^{-\frac{1}{2}}-1+\chi_{Y},
$$

together with the fact that $\alpha^{(2)}\left(\chi_{Y}\right)=\chi_{Y} \otimes 1$.

Denote the category of compact quantum groups acting on the above spectral triple on a finite metric space $(X, d)$ with action $\alpha$ satisfying $\alpha^{(2)}(d)=d \otimes 1$ by $\mathbf{C}_{\mathbf{X}, \mathbf{d}}^{\text {Ban }}$. We want to show that the universal object in $\mathbf{C}_{\mathbf{X}, \mathbf{d}}^{\text {Ban }}$, say QISO ${ }^{\text {Ban }}$ (which is shown to exist in $[\mathrm{Ban}]$ ), is isomorphic to the quantum group $\mathrm{QISO}_{I}^{+}(D)$. The proof of this fact is contained in the following two lemmas. Recall that we always assume that $(X, d)$ is a finite metric space.

Lemma 4.3. Let $(\mathrm{S}, \alpha)$ be a quantum isometry of $(X, d)$ in the sense of Banica, i.e. an object in $\mathbf{C}_{\mathbf{X}, \mathbf{d}}^{\mathrm{Ban}}$. Then there is a unitary representation $U$ of $\mathbf{S}$ on $\mathrm{H}$ such that $(\mathrm{S}, U) \in \operatorname{Obj}\left(\mathbf{C}_{\mathbf{X}, \mathbf{d}}^{(\mathbf{2})}\right)$, with $\alpha_{U}=\alpha$ on $C(X)$.

Proof. Define $\widetilde{U}=I_{\mathbb{C}^{2}} \otimes \widetilde{\alpha^{(2)}}$ on $\mathcal{B}(\mathrm{H}) \otimes \mathrm{S}$. Then $\widetilde{U}$ gives a unitary representation since $\widetilde{\alpha^{(2)}}$ is one.

Moreover, recalling from Lemma 4.1 that for all $f \in C(X)$ one has $\operatorname{Tr}(\pi(f))=$ $(2 n-1) \tau_{0}(f)$ and that $\alpha$ preserves $\tau_{0}$, we immediately observe that the action $\alpha_{U}$ preserves the volume form corresponding to $R=I$. Note that for any $X \in M_{2}(\mathbb{C})$,

$$
\widetilde{U}\left(X \otimes M_{\phi} \otimes I_{\mathrm{S}}\right) \widetilde{U}^{-1}=X \otimes \widetilde{\alpha^{(2)}}\left(M_{\phi} \otimes I\right) \widetilde{\alpha^{(2)}}-1=X \widetilde{M_{\alpha^{(2)}(\phi)}},
$$

where $\phi=s^{*}(f)$, and for $Y=y_{1} \otimes y_{2} \in C(Y) \otimes \mathrm{S}, \widetilde{M}_{Y}$ denotes $M_{y_{1}} \otimes y_{2}$.

$$
\widetilde{U}(D \otimes I) \widetilde{U}^{-1}=\left(\begin{array}{cc}
0 & i \\
-i & 0
\end{array}\right) \otimes \widetilde{M_{\alpha^{(2)}\left(d^{-1}\right)}}=\left(\begin{array}{cc}
0 & i \\
-i & 0
\end{array}\right) \otimes M_{d^{-1}} \otimes I=D \otimes I,
$$


using $\alpha^{(2)}\left(d^{-1}\right)=d^{-1} \otimes 1$ and Banica's condition from Lemma 4.2. This shows that $U$ commutes with $D$. Further it is easy to see that for any $f \in C(X)$,

$$
\begin{aligned}
\widetilde{U} & \left(\pi^{(2)}(f) \otimes 1\right) \widetilde{U}^{-1} \\
& =\widetilde{U}\left(\pi_{1}^{(2)}(f) \otimes 1\right) \widetilde{U}^{-1}+\widetilde{U}\left(\pi_{2}^{(2)}(f) \otimes 1\right) \widetilde{U}^{-1} \\
& =\widetilde{U}\left(\left(\begin{array}{ll}
1 & 0 \\
0 & 0
\end{array}\right) \otimes s^{*}(f) \otimes 1\right) \widetilde{U}^{-1}+\widetilde{U}\left(\left(\begin{array}{ll}
0 & 0 \\
0 & 0
\end{array}\right) \otimes t^{*}(f) \otimes 1\right) \widetilde{U}^{-1} \\
& =\left(\begin{array}{ll}
1 & 0 \\
0 & 0
\end{array}\right) \otimes M_{\alpha(2)}\left(s^{*} f\right) \\
& =\left(\begin{array}{ll}
1 & 0 \\
0 & 0
\end{array}\right) \otimes s^{*}\left(f_{(1)}\right) \otimes f_{(2)} \\
& =(\pi \otimes \mathrm{id}) \alpha(f) \subseteq \pi(C(X)) \otimes \mathrm{S} .
\end{aligned}
$$

Note that in the above we have used the Sweedler notation $\alpha(f)=f_{(1)} \otimes f_{(2)}$. This implies that $(\mathrm{S}, U) \in \operatorname{Obj}\left(\mathbf{C}_{\mathbf{X}, \mathbf{d}}^{(\mathbf{2})}\right)$. It is obvious from the construction that $\alpha_{U}=\alpha$.

Lemma 4.4. Let $(\widetilde{\mathrm{S}}, U) \in \operatorname{Obj}\left(\mathbf{C}_{\mathbf{X}, \mathbf{d}}^{(\mathbf{2})}\right)$, with $\mathrm{S}$ being the largest Woronowicz $C^{*}$ subalgebra of $\widetilde{\mathrm{S}}$ such that the action $\alpha_{U}$ maps $C(X)$ into $C(X) \otimes \mathrm{S}$. Then $\left(\mathrm{S}, \alpha_{U}\right)$ is an object of $\mathbf{C}_{\mathbf{X}, \mathbf{d}}^{\mathbf{B a n}}$.

Proof. The fact that $U$ commutes with $D$ implies that $U$ commutes with $D^{2}=$ $I_{\mathbb{C}^{2}} \otimes M_{c}$ on $\mathbb{C}^{2} \otimes l^{2}(Y)$. Since $U=I \otimes \alpha^{(2)}$, it follows that $\alpha^{(2)}(c)=c \otimes 1$; hence (by Lemma 4.2) $(\mathrm{S}, \alpha)$ is a quantum isometry in the sense of Banica.

Lemma 4.2. Lemma 4.3 and Lemma 4.4 put together imply immediately the following:

Theorem 4.5. We have the following isomorphisms of compact quantum groups:

$$
\mathrm{QISO}^{\mathrm{Ban}}(X) \cong \mathrm{QISO}_{I}^{+}(D) \cong \mathrm{QISO}^{\mathcal{L}}(X) \text {. }
$$

Remark 4.6. We can accommodate graphs in the framework of the above theorem if we view a finite nondirected graph $(V, E)$ as a metric space $\left(V, d_{E}\right)$, where

$$
d_{E}(v, w)=1 \text { if }(v, w) \in E, \quad d_{E}(v, w)=\infty \text { if }(v, w) \notin E
$$

$(v, w \in V, v \neq w)$. A similar observation was already made in Ban. Here Theorem 4.5] shows that quantum symmetry groups of finite graphs of [Bic] can be viewed as quantum isometry groups associated to the natural Laplacians on such graphs.

\section{REMARKS ON QUANTUM ISOMETRIES OF ARBITRARY METRIC SPACES}

In view of Lemma 4.2 it is reasonable to define a 'quantum isometry' of a general metric space $(X, d)$ to be a (faithful) action $\alpha$ on $C(X)$ by a compact quantum group $S$ such that $\alpha^{(2)}(d)=d \otimes 1$ (here again the metric $d$ is viewed as an element of $C(X \times X))$. It is however a nontrivial (and open) problem to see whether there exist any universal objects in the category of such quantum isometries of $(X, d)$ when $X$ is not a finite set. We shall take up this issue elsewhere, but would like to conclude this article with some computations for two simple yet interesting examples, namely the unit interval $[0,1]$ and the circle $S^{1}$ (both equipped with the usual Euclidean metric). 
$\mathbf{X}=[\mathbf{0}, \mathbf{1}]$. The $C^{*}$-algebra $C(X)$ is the universal unital $C^{*}$-algebra generated by a selfadjoint operator $T$ satisfying $0 \leq T \leq 1$. The metric is given by $\left(x_{1}, x_{2} \in[0,1]\right)$

$$
d^{2}\left(x_{1}, x_{2}\right)=x_{1}{ }^{2}-2 x_{1} x_{2}+x_{2}{ }^{2} .
$$

Thus, as an element of $C([0,1]) \otimes C([0,1])$, the metric $d$ is given by $d^{2}=T^{2} \otimes 1-$ $2 T \otimes T+1 \otimes T^{2}$. Given a quantum isometric action $\alpha$ of a c.q.g. S, let us write

$$
\alpha(T)=\sum_{n \geq 0} T^{n} \otimes q_{n}
$$

(the series is strongly convergent). Since $T$ is selfadjoint and $\alpha$ is a *-homomorphism, each $q_{n}$ is also selfadjoint. Comparing coefficients of $T^{n} \otimes T^{n}$ (for $n \geq 2$ ) and $T^{2} \otimes 1$ in the equation $\alpha^{(2)}\left(d^{2}\right)=d^{2} \otimes 1$, we get (respectively)

$$
q_{n}^{2}=0, \quad n \geq 2
$$

and

$$
q_{2} q_{0}+q_{0} q_{2}+q_{1} q_{1}-2 q_{2} q_{0}=1 \text {. }
$$

Since each $q_{n}$ is selfadjoint, the first equation above implies immediately that

$$
q_{n}=0, \quad n \geq 2 .
$$

Using $q_{2}=0$ in the second equation we deduce that $q_{1}^{2}=1$. Thus, $q_{1}$ is a reflection (selfadjoint unitary) and as such can be written as $q_{1}=P-P^{\perp}$, where $P$ is an orthogonal projection.

Moreover, $\alpha(T)$ is a positive contraction as $T$ is so, which implies that

$$
0 \leq 1 \otimes q_{0}+T \otimes q_{1} \leq 1 .
$$

By applying to both sides of the above inequality the evaluation functional at a point $t \in[0,1]$, we obtain

$$
0 \leq q_{0}+t q_{1} \leq 1, \quad t \in[0,1] .
$$

Putting $t=0$ we note that $q_{0}$ is a positive contraction. Further multiplying by $P$ both sides of (5.1) we obtain

$$
0 \leq P q_{0} P+t P \leq P, \quad t \in[0,1] .
$$

Putting $t=1$ yields $P q_{0} P=0$, which implies that $q_{0}$ maps $P$ to $P^{\perp}$ (here and below we identify $P$ and $P^{\perp}$ with the corresponding subspaces of the Hilbert space). Similarly, multiplying both sides of (5.1) by $P^{\perp}$ we obtain

$$
0 \leq P^{\perp} q_{0} P^{\perp}-t P^{\perp} \leq P^{\perp}, \quad t \in[0,1] .
$$

Putting $t=0$ and $t=1$, we get (respectively)

$$
P^{\perp} q_{0} P^{\perp} \leq P^{\perp}
$$

and

$$
P^{\perp} q_{0} P^{\perp} \geq P^{\perp} .
$$

It follows that $P^{\perp} q_{0} P^{\perp}=P^{\perp}$; hence $q_{0}: P^{\perp} \rightarrow P^{\perp}$ and $\left.q_{0}\right|_{P^{\perp}}=I$. Recalling that $q_{0}$ is a contraction and using the above two observations we conclude that $q_{0}=P^{\perp}$.

Thus, $\alpha(T)=1 \otimes P^{\perp}+T \otimes\left(P-P^{\perp}\right)$, which clearly implies (by faithfulness of $\alpha$ ) that $\mathrm{S}=C^{*}(P)$ is commutative; i.e. there is no 'quantum isometry' of $X$. 
$\mathbf{X}=\mathbf{S}^{\mathbf{1}}$. The $C^{*}$-algebra $C\left(S^{1}\right)$ is the universal unital $C^{*}$-algebra generated by a unitary, say $Z$. The metric is given this time by $\left(z_{1}, z_{2} \in S^{1}\right)$

$$
d^{2}\left(z_{1}, z_{2}\right)=2-z_{1} \overline{z_{2}}-z_{2} \overline{z_{1}},
$$

so in the tensor picture we get $d^{2}=2 \otimes 1-Z \otimes Z^{*}-Z^{*} \otimes Z$.

Let $\mathrm{S}$ be a c.q.g. with an isometric action $\alpha$ on $S^{1}$. Let

$$
\alpha(Z)=\sum_{n=0}^{\infty} Z^{n} \otimes q_{n}+\sum_{n=1}^{\infty} Z^{* n} \otimes q_{n}^{\prime}
$$

where $q_{n}, q_{n}^{\prime} \in \mathrm{S}$. Comparing the coefficients of $1 \otimes 1$ and $Z^{n} \otimes Z^{* n}$ for $n \geq 2$ on both sides of the equation $\alpha^{(2)}\left(d^{2}\right)=d^{2} \otimes 1$ we get this time

$$
q_{0} q_{0}^{*}+q_{0}^{*} q_{0}=0, \quad q_{n} q_{n}^{*}+q_{n}^{\prime *} q_{n}^{\prime}=0, \quad n \geq 2 .
$$

This proves that $q_{0}=0$ and $q_{n}=q_{n}^{\prime}=0$ for all $n \geq 2$. Thus, $\alpha(Z)=Z \otimes q_{1}+Z^{*} \otimes q_{1}^{\prime}$; i.e., $\alpha$ is 'linear' as in subsection 2.2 in $\mathrm{BG}_{1}$ and hence by Theorem 2.4 of that paper, $\mathrm{S}$ must be commutative as a $C^{*}$-algebra and so a quantum subgroup of $C(I S O(X))=C\left(S^{1} \rtimes Z_{2}\right)$. Then one can conclude that the investigated quantum isometry group $Q I S O\left(S^{1}, d\right)$ is equal to $C\left(S^{1} \rtimes \triangleleft Z_{2}\right)$, which can be interpreted as a statement that there is no 'quantum isometry' of $S^{1}$ viewed as a metric space. We remark that we arrived at a similar conclusion in $\mathrm{BG}_{1}$ and $\mathrm{Gos}$ by viewing $S^{1}$ as a Riemannian manifold. So, the observation made in the present paper in some sense strengthens the results of $\mathrm{BG}_{1}$ and $\mathrm{Gos}$ about the quantum actions on $S^{1}$.

\section{ACKNOWLEDGMENT}

The main results of this paper were obtained during the visit of the third named author to the Indian Statistical Institute in Kolkata, which was made possible thanks to the support of the UKIERI project Quantum Probability, Noncommutative Geometry and Quantum Information.

The first and second named authors would like to acknowledge support from the National Board of Higher Mathematics, D.A.E. (India) and the Indian National Science Academy, respectively.

\section{REFERENCES}

[Ban] T. Banica, Quantum automorphism groups of small metric spaces, Pacific J. Math. 219 (2005), no. 1, 27-51. MR2174219 (2006h:16054)

$\left[\mathrm{BG}_{1}\right]$ J. Bhowmick and D. Goswami, Quantum Isometry Groups: Examples and Computations, Comm. Math. Phys. 285 (2009), no. 2, 421-444. MR2461983

$\left[\mathrm{BG}_{2}\right]$ J. Bhowmick and D. Goswami, Quantum group of orientation-preserving Riemannian Isometries, preprint available at arXiv:0806.368\%.

[Bic] J.Bichon, Quantum automorphism groups of finite graphs, Proc. Amer. Math. Soc. 131 (2003), no. 3, 665-673. MR1937403 (2003j:16049)

[Cha] P.S. Chakraborty, $C^{*}$-algebra generated by projections, preprint available at arXiv:0306064.

$\left[\mathrm{CI}_{1}\right] \quad$ E. Christensen and C. Ivan, Spectral triples for AF $C^{*}$-algebras and metrics on the Cantor set, J. Operator Theory 56 (2006), no. 1, 17-46. MR2261610 (2008j:46057)

$\left[\mathrm{CI}_{2}\right]$ E. Christensen and C. Ivan, Sums of two dimensional spectral triples, Math. Scand. 100 (2007), no. 1, 35-60. MR2327719 (2009a:58030)

$\left[\mathrm{Co}_{1}\right]$ A. Connes, Compact metric spaces, Fredholm modules, and hyperfiniteness, Ergodic Theory Dynam. Systems 9 (1989), no. 2, 207-220. MR1007407(90i:46124) 
$\left[\mathrm{Co}_{2}\right]$ A. Connes, "Noncommutative Geometry", Academic Press, London-New York, 1994. MR.1303779 (95j:46063)

[Gos] D. Goswami, Quantum Group of Isometries in Classical and Noncommutative Geometry, Comm. Math. Phys. 285 (2009), no. 1, 141-160. MR2453592

[Lan] C.E. Lance, "Hilbert $C^{*}$-modules. A toolkit for operator algebraists," London Mathematical Society Lecture Note Series, 210. Cambridge University Press, Cambridge, 1995. MR 1325694 (96k:46100)

[Rie] M. Rieffel, Metrics on state spaces, Documents Math. 4 (1999), 559-600. MR1727499 (2001g:46154)

[Wan] S. Wang, Quantum symmetry groups of finite spaces, Comm. Math. Phys. 195 (1998), no. 1, 195-211. MR.1637425 (99h:58014)

Stat-Math Unit, Indian Statistical Institute, 203, B. T. Road, Kolkata 700 208, India

E-mail address: jyotish $r @ i s i c a l . a c . i n$

Current address: ICTP, Mathematics Section, Strada Costiera 11, I-34151, Trieste, Italy

E-mail address: jbhowmic@ictp.it

Stat-Math Unit, Indian Statistical Institute, 203, B. T. Road, Kolkata 700 208, India

E-mail address: goswamid@isical.ac.in

Department of Mathematics, University of Łódź, ul. Banacha 22, 90-238 Łódź, POLAND

Current address: Department of Mathematics and Statistics, Lancaster University, Lancaster, LA1 4YF, United Kingdom

E-mail address: a.skalski@lancaster.ac.uk 